\title{
A Plasmonic Fluid with Dynamically Tunable Optical Properties
}

\author{
Rama Ranjan Bhattacharjee, ${ }^{a}$ Ruipeng $\mathrm{Li}^{b},{ }^{b}$ Luis Estevez, ${ }^{a}$ \\ Detlef-M. Smilgies, ${ }^{c}$ Aram Amassian ${ }^{b}$ and Emmanuel P. Giannelis ${ }^{* a}$
}

${ }^{a}$ Materials Science \& Engineering, Cornell University, Ithaca, New York 14853, USA

*EMAIL: epg2@cornell.edu, Fax No.: +1 607-255-2365

${ }^{b}$ King Abdullah University of Science and Technology, Materials Science and Engineering, Jeddah, Kingdom of Saudi Arabia.

${ }^{c}$ Cornell High-Energy Synchrotron Source (CHESS), Cornell University, Ithaca, New York 14853, USA

$\dagger$ Electronic supplementary information (ESI) available: (A) Thermogravimetric analysis (TGA) data

for samples 1 \& 2. (B) Video of dynamic color changes observed while shearing sample 1. (C) Video of dynamic spectral changes observed while shearing sample $\mathbf{1}$. 
ABSTRACT

We report the first synthesis of a gold nanorod (GNR)-based nanocomposite that exhibits solid-like plasmonic properties while behaving in a liquid-like manner. Tuning the degree of GNR clustering controls the material's responsiveness to external stimuli, such as mechanical shearing, due to the sensitivity of the localized surface plasmon resonance to interparticle interactions. 
Recently, one dimensional nanoparticles have been shown to provide building blocks for materials applications due to their intriguing optical and electronic properties. ${ }^{1-3}$ Gold nanorods (GNRs) for example, have interesting optical properties due to the collective oscillation of conduction electrons in different directions within the particles upon interaction with light. ${ }^{2-6}$ The energy levels of the resulting localized surface plasmon resonance (LSPR) bands depend on the size and average aspect ratio of the particles $^{3,7}$ and can be tuned by controlling the interparticle separation and relative orientation of the GNRs. ${ }^{8,9}$ In solution, surface functionalized GNRs have been assembled into various geometries by changing $\mathrm{pH},{ }^{10}$ hydrophilicity ${ }^{11,12}$ and by recognition techniques. ${ }^{13,14}$ These assemblies show interesting optical properties which can be used in surface enhanced Raman scattering, ${ }^{15,} 16$ bioimaging, ${ }^{17}$ biosensor, ${ }^{16}$ and cancer therapy. ${ }^{15-18}$ In the solid state, there are reports of assembled and oriented GNRs in polymers or porous supports that show polarization dependent optical properties. ${ }^{9,}{ }^{19}$ However, GNR fluids with tunable viscoelastic and plasmonic properties, i.e., between the liquid and solid states, have not been reported in literature, until now. We report the first solventless GNR-based nanocomposite fluid with tunable plasmonic and viscoelastic properties. Mechanical shearing of these fluids can produce dynamical and reversible spectral and chromatic changes when GNRs are arranged into clusters. These unique properties result from a combination of solid-like assembly of GNR inclusions and a fluid-like behavior of the matrix. This novel material system based on one-dimensional metallic nanoparticles may pave the way for new applications in the fields of biology, micro/nanofluidics, sensing, plasmonics, and beyond.

We have recently focused on a new, tunable materials platform prepared by stepwise functionalization of nanoparticles (NP), which results in fluidities ranging from simple Newtonian liquids to gels and solids having interesting properties. ${ }^{20-26}$ These are generically called nanoscale ionic materials (NIMs), and are a new and unique class of hybrid organic-inorganic composites consisting of an inorganic nanoparticle core, a charged corona, and a counterion canopy. Each NP in the composite therefore carries its own canopy via primary ionic bonds, and so the ensemble behaves like a viscoelastic fluid. The synthesis strategy was modified slightly for the GNR NIMs to address specific 
challenges associated with the surface modification of GNRs. In particular, Dai et al. have shown that the CTAB bi-layer often results in irreversible aggregation of the nanorods, if surface modification is attempted. ${ }^{27}$ Hence, our approach towards the synthesis of the fluidic-GNRs was to consecutively build up a layer of sulfonic acid groups (part of the corona) on the surface of the GNRs, thus keeping the CTAB bilayer stable, and then titrating the sulfonic groups with a polyethylene glycol oligomer that is end-terminated with an amine group (Jeffamine M2070). The entire synthetic approach is schematically shown in Scheme 1. A layer by layer approach was adopted with polystyrene sulfonate (PSS) selfassembled on the CTAB covered GNRs. ${ }^{17}$ Excess CTAB was washed out by centrifugation (sample 1) or dialysis (sample 2), resulting in samples with different nanoparticle arrangements (see below). The sulfonic groups of PSS were protonated by ion exchange using an ion-exchange resin to $\mathrm{pH} \sim 2.7$ and then titrated with the jeffamine to $\mathrm{pH} \sim 4.8$ to form the canopy. ${ }^{24}$ The synthesis process can be termed 'green' as all reactions are done in water and do not involve any toxic or harmful chemicals. The end product is an intensely colored fluid salt (GNR-NIMS) that is isolated by freeze drying (shown in inset of Scheme 1).

Transmission electron micrographs (TEM) of samples $\mathbf{1}$ and $\mathbf{2}$ deposited on a TEM grid are shown in Figure 1. Sample 1 reveals small GNR clusters whereas sample 2 consists of well-dispersed GNRs. In both cases, TEM images reveal a relatively narrow spread of GNR diameters (lengths), with a mean value of $12.8 \mathrm{~nm}(49.4 \mathrm{~nm})$ and a standard deviation of $2.9 \mathrm{~nm}(6.8 \mathrm{~nm})$. We calculate a mean aspect ratio of $\sim 4.0$ with a standard deviation of 0.6. The presence and importance of interparticle interactions can be probed via small-angle X-ray scattering (SAXS). In Figure 2(a), we compare SAXS measurements taken in transmission mode for samples $\mathbf{1}$ and $\mathbf{2}$. Correlation peaks seen in the case of sample 1 confirm that most GNRs in the sample are in close proximity with other GNRs, in agreement with TEM. By contrast, GNRs in sample 2 tend to be isolated or in small clusters (e.g., dimers, trimers), as evidenced by the absence of a correlation peak. Quantitative analysis of SAXS data for sample 1 reveals a mean interparticle spacing (center-to-center) of $20.9 \mathrm{~nm}$, with a standard deviation of $2.1 \mathrm{~nm}$, indicating that GNRs are assembled predominantly in a side-by-side manner, since the mean diameter is 
$12.8 \mathrm{~nm}$. In such an arrangement, the mean surface-to-surface separation between two adjacent GNRs in a side-by-side arrangement is approximately $8.1 \mathrm{~nm}$. This separation is much smaller than 2.5 times the diameter of GNRs. This means the plasmonic response of sample $\mathbf{1}$ is expected to depend sensitively upon interparticle interactions. ${ }^{8,31-33}$

A small drop of each fluid was placed in between two microscope slides and monitored in an unpolarized optical microscope. Using an electrical motor, the top microscope slide was translated, resulting in mechanical shearing of the fluid film. This caused an immediate and distinctive color change in sample 1 from violet to red ( $c f$. Fig. 2), while no change was observed in the case of sample 2 in similar circumstances. Upon stopping the motor, sample 1 reverted to its original color. Repetitive shearing of sample $\mathbf{1}$ yielded the same color change and the same recovery behavior, indicating a remarkably reversible phenomenon and a lack of memory by the sample.

A more in-depth investigation of the optical response and its dynamic changes were performed using static and time-resolved transmission spectrophotometry, respectively (cf. Fig. 2). In static mode, sample 2 exhibits two distinct bands at $\lambda=650$ and $850 \mathrm{~nm}$, which is very much similar to reports of GNR suspension with similarly high aspect ratio. These correspond to the oscillation of the free electrons along (longitudinal; low energy) and perpendicular to (transverse; high energy) the long axis of the rods. ${ }^{28-30}$ By contrast, sample 1 exhibits a single broad absorption dip at $\lambda=540 \mathrm{~nm}$ with a shoulder at longer wavelengths, indicating the presence of a second absorption peak nearby. The optical response of sample 1 differs from most other reports of absorption by GNR suspensions with an aspect ratio of $\sim 3$ and above, because of the presence of only a single absorption band.

Upon shearing sample 1, we observe an instantaneous spectral shift, consisting in splitting of the absorption bands into two distinct peaks. The low-energy peak undergoes a significant red shift up to $\lambda$ $=690 \mathrm{~nm}$ (see video of dynamic spectral and color changes in ESI), while the high energy peak is blue shifted to $\lambda=540 \mathrm{~nm}$. The spectrum of sample 1 is more familiar when it is sheared, because of the separation of the two absorption bands. When shearing is stopped, the spectrum slowly reverts back to 
its original state via an exponential decay with a characteristic time scale of $\sim 440$ seconds, becoming indistinguishable from the initial spectrum of the sample after $\sim 30$ minutes. Sample 2 does not exhibit any visible spectral changes when subjected to mechanical shearing, in agreement with optical observations.

To explain the differences between the optical properties of samples $\mathbf{1}$ and $\mathbf{2}$ in static mode, we first note that the mean size, aspect ratio and size distribution of GNRs are identical in both samples as these were prepared from the same batch of GNRs; thermogravimetric measurements (see ESI) also show that the core-to-canopy ratio is the same in both samples. Differences in the degree of clustering and interaction between GNRs are therefore the only plausible explanation for these observations. Localized surface plasmon resonance interactions between GNRs have been described by the plasmon hybridization model, an electromagnetic analogue to the molecular orbital theory, whereby interactions between the nanoparticles give rise to different energy levels and change the spectroscopic properties of the nanocomposite. ${ }^{8,} 34$ Coupling of the GNR longitudinal modes leads to bonding (attractive) and antibonding (repulsive) interactions when dimers are respectively arranged side-by-side or end-to-end, ${ }^{33,}$ ${ }^{35,36}$ resulting in a decrease (red shift) or increase (blue shift) of the energy of the absorption band.

In the case of sample 1, close-packed clusters of GNRs typically contain a mixture of side-by-side and end-to-end arrangements. The former tend to be more common; e.g., GNRs experience twice as many side-by-side interactions than end-to-end ones in a hexagonal arrangement. It is not surprising then to find the longitudinal band of sample $\mathbf{1}$ to be blue shifted relative to sample 2 .

Upon shearing sample 1, the longitudinal band undergoes an important red shift. This behavior is absent in sample 2, clearly pointing to interparticle interactions in the GNR clusters as the main cause. For further insight, we have performed time-resolved SAXS measurements in transmission mode on sample 1 in the relaxed and sheared states (figure 3). SAXS images clearly reveal a uniform ring in the relaxed state, which corresponds to the first correlation peak and confirms the isotropy of the sample. Upon shearing (left to right), we observe the loss of lateral correlation along the direction of shearing as indicated by the ring becoming partial. The azimuthal intensity plot reveals an increase (decrease) of 
scattering intensity in the in-plane direction perpendicular (parallel) to shearing. Comparison between the relaxed and sheared states of the sample in terms of the radial position of the correlation ring and its azimuthal intensity profile leads to four key observations: (1) the intensity of the correlation decreases in the direction parallel to shearing; (2) the intensity of the correlation increases in the direction normal to shearing; (3) the radial position of the ring does not appear to change; and (4) the total scattering intensity of the sample remains unchanged.

These observations demonstrate that shearing leads to a net change in the orientation of GNR clusters, whereby the long axis of GNRs becomes parallel to the direction of shearing. However, this occurs without a noticeable change in the size of the clusters or in the mean side-by-side interparticle spacing. In Figure 3, we also show cross-polarized microscopy images of sample $\mathbf{1}$ during shearing. Here, a dark image indicates an isotropic sample with randomly oriented GNRs and GNR clusters; a bright image reveals an optically - and by extension a structurally - anisotropic material. All samples are dark before shearing and only sample 1 changes color to bright red during shearing. The sample returns to its initial state after shearing (not shown). The color change observed in cross-polarized microscopy during shearing is consistent with the increased transmittance in the red part of the spectrum; it proves that the macroscopic color of the sample and its spectral changes are somehow related to the anisotropic alignment of the GNRs along the direction of mechanical shearing.

However, these results do not directly explain the red shift in the position of the longitudinal peak. In fact, Perez-Juste et al. have previously shown that aligning isolated GNRs in a polymer matrix does not result in a shift of the spectral position of the longitudinal peak, only in a change in its intensity. ${ }^{19}$ Instead, evidence points decisively toward GNR clustering as the cause of differences between samples 1 and 2. Indeed, clustering makes the optical properties of sample $\mathbf{1}$ hypersensitive to small changes in the localized surface plasmon resonance, especially due to perturbations to interparticle interactions. This may be the key reason for (i) spectral differences between samples $\mathbf{1}$ and $\mathbf{2}$ in the static mode and (ii) to the sensitivity of sample 1 to shearing. The shearing-induced rotation and alignment of the anisotropic GNR clusters along the direction of shearing - as seen by SAXS measurements - can cause 
relative sliding of GNRs, as well as stretching and/or compression along the long axis (end-to-end bonds) - which are not easy to detect by SAXS measurements. These have been shown to dramatically influence the LSPR interactions, ${ }^{8}$ and may be playing a key role in dictating changes in the optical properties of sample 1. Further insight into the mechanisms responsible for the color change will require additional experiments and computer simulations which are beyond the scope of this report.

In summary, we have successfully synthesized for the first time a new class of functional GNR nanocomposites, which combine flow behavior with the well-known surface plasmon resonance properties of GNRs. The new nanocomposite fluids are synthesized by a layer-by-layer deposition of sulfonated polystyrene on CTAB GNRs followed by neutralization using an amine terminated oligomeric ethylene glycol. The response of the plasmonic solventless fluid can be tuned by engineering the interparticle interactions via assembly of GNRs within the fluid. The unique liquid-like properties of the nanocomposite allows it to flow at room temperature and make it responsive to external stimuli, such as mechanical shearing. The plasmonic response is fully reversible and is due to the formation of large GNR clusters in the nanocomposite fluid. This new class of material might pave new applications in plasmonics, micro/nanofluidics, sensing, and biology,

\section{Acknowledgements}

We would like to acknowledge Prof. Lynden Archer for helpful discussions. This work was made possible by Award No. KUS-C1-018-02 made by King Abdullah University of Science and Technology (KAUST). We acknowledge use of facilities at the Cornell Center for Materials Research, a National

Science Foundation Materials Research Science and Engineering Center (NSF DMR-0520404), and the Cornell High Energy Synchrotron Source, also supported by the National Science Foundation and NIHNIGMS (NSF DMR-0225180). 


\section{Figures and Legends}

Scheme 1. Synthesis of a gold nanorod-based solventless fluid by step-wise surface modification. Inset shows an image of the sample after freeze-drying.

Figure 1. TEM and SAXS data of samples 1 (left) \& 2 (right). Scale bar $=500 \mathrm{~nm}$. Top right insets show enlarged views of the GNRs. Scale bar $=200 \mathrm{~nm}$. Overlays show the corresponding transmission SAXS spectra.

Figure 2. Dynamic spectral changes observed in the relaxed state and while shearing samples 1 (topleft) \& 2 (bottom-left). Inset shows time-evolution of the integrated transmittance (660 nm $\leq \lambda \leq 750$ $\mathrm{nm}$ ) from the moment immediately before shearing, until the sample is returns to its relaxed state. Images shown on top-right and bottom-right represent non-polarized optical microscopy snapshots taken in the relaxed and sheared states of samples $1 \& 2$ respectively. Videos showing the dynamic changes of transmission spectrum and color upon shearing are shown in ESI.

Figure 3. SAXS images of the sample 1 in the relaxed state (A) and while shearing (B). SAXS data showing the variation of intensity along the azimuthal angle before and during shearing (bottom left). Cross polarization image of sample 1 taken while shearing (bottom right) and after relaxing (Inset). 


\section{Notes and References}

1. $\quad$ L. C. Palmer and S. I. Stupp, Acc. Chem. Res., 2008, 41, 1674-1684.

2. M. A. El-Sayed, Acc. Chem. Res., 2001, 34, 257-264.

3. C. Burda, X. B. Chen, R. Narayanan and M. A. El-Sayed, Chem. Rev., 2005, 105, 1025-1102.

4. M. C. Daniel and D. Astruc, Chem. Rev., 2004, 104, 293-346.

5. K. L. Kelly, E. Coronado, L. L. Zhao and G. C. Schatz, J. Phys. Chem. B, 2003, 107, 668-677.

6. Y. N. Xia and N. J. Halas, MRS Bull., 2005, 30, 338-344.

7. M. B. Mohamed, V. Volkov, S. Link and M. A. El-Sayed, Chem. Phys. Lett., 2000, 317, $517-$ 523.

8. A. M. Funston, C. Novo, T. J. Davis and P. Mulvaney, Nano Lett., 2009, 9, 1651-1658.

9. G. A. Wurtz, W. Dickson, D. O'Connor, R. Atkinson, W. Hendren, P. Evans, R. Pollard and A. V. Zayats, Opt. Express, 2008, 16, 7460-7470.

10. Z. H. Sun, W. H. Ni, Z. Yang, X. S. Kou, L. Li and J. F. Wang, Small, 2008, 4, 1287-1292.

11. W. S. Chang, L. S. Slaughter, B. P. Khanal, P. Manna, E. R. Zubarev and S. Link, Nano Lett., 2009, 9, 1152-1157.

12. Z. H. Nie, D. Fava, E. Kumacheva, S. Zou, G. C. Walker and M. Rubinstein, Nat. Mater., 2007, 6, 609-614.

13. H. Nakashima, K. Furukawa, Y. Kashimura and K. Torimitsu, Chem. Commun., 2007, 10801082.

14. K. K. Caswell, J. N. Wilson, U. H. F. Bunz and C. J. Murphy, J. Am. Chem. Soc., 2003, 125, 13914-13915.

15. X. G. Hu, W. L. Cheng, T. Wang, Y. L. Wang, E. K. Wang and S. J. Dong, J. Phys. Chem. B, 2005, 109, 19385-19389. 
16. R. A. Tripp, R. A. Dluhy and Y. P. Zhao, Nano Today, 2008, 3, 31-37.

17. P. N. Sisco, C. G. Wilson, E. Mironova, S. C. Baxter, C. J. Murphy and E. C. Goldsmith, Nano Lett., 2008, 8, 3409-3412.

18. T. S. Hauck, T. L. Jennings, T. Yatsenko, J. C. Kumaradas and W. C. W. Chan, Adv. Mater., 2008, 20, 3832-3838.

19. J. Perez-Juste, B. Rodriguez-Gonzalez, P. Mulvaney and L. M. Liz-Marzan, Adv. Func. Mater., 2005, 15, 1065-1071.

20. A. B. Bourlinos, E. P. Giannelis, Q. Zhang, L. A. Archer, G. Floudas and G. Fytas, Eur. Phys. J. E, 2006, 20, 109-117.

21. A. B. Bourlinos, A. Stassinopoulos, D. Anglos, R. Herrera, S. H. Anastasiadis, D. Petridis and E. P. Giannelis, Small, 2006, 2, 513-516.

22. A. B. Bourlinos, V. Georgakilas, V. Tzitzios, N. Boukos, R. Herrera and E. R. Giannelis, Small, 2006, 2, 1188-1191.

23. A. B. Bourlinos, R. Herrera, N. Chalkias, D. D. Jiang, Q. Zhang, L. A. Archer and E. P. Giannelis, Adv. Mater., 2005, 17, 234-237.

24. R. Rodriguez, R. Herrera, L. A. Archer and E. P. Giannelis, Adv. Mater., 2008, 20, 4353-4358.

25. A. A. Voevodin, R. A. Vaia, S. T. Patton, S. Diamanti, M. Pender, M. Yoonessi, J. Brubaker, J. J. Hu, J. H. Sanders, B. S. Phillips and R. I. MacCuspie, Small, 2007, 3, 1957-1963.

26. S. T. Patton, A. A. Voevodin, R. A. Vaia, M. Pender, S. J. Dianianti and B. Phillips, J. Microelectromech. Sys., 2008, 17, 741-746.

27. Q. Dai, J. Coutts, J. H. Zou and Q. Huo, Chem. Commun., 2008, 2858-2860.

28. M. B. Mohamed, K. Z. Ismail, S. Link and M. A. El-Sayed, J. Phys. Chem. B, 1998, 102, 93709374.

29. Y. Y. Yu, S. S. Chang, C. L. Lee and C. R. C. Wang, J. Phys. Chem. B, 1997, 101, 6661-6664.

30. G. C. Papavassiliou, Prog. Solid State Chem., 1979, 12, 185-271. 
31. C. Sonnichsen, B. M. Reinhard, J. Liphardt and A. P. Alivisatos, Nat. Biotechnol., 2005, 23, $741-745$.

32. B. M. Reinhard, S. Sheikholeslami, A. Mastroianni, A. P. Alivisatos and J. Liphardt, PNAS, 2007, 104, 2667-2672.

33. B. M. Reinhard, M. Siu, H. Agarwal, A. P. Alivisatos and J. Liphardt, Nano Lett., 2005, 5, 22462252.

34. S. Eustis and M. El-Sayed, J. Phys. Chem. B, 2005, 109, 16350-16356.

35. E. Prodan, C. Radloff, N. J. Halas and P. Nordlander, Science, 2003, 302, 419-422.

36. P. Nordlander, C. Oubre, E. Prodan, K. Li and M. I. Stockman, Nano Lett., 2004, 4, 899-903. 

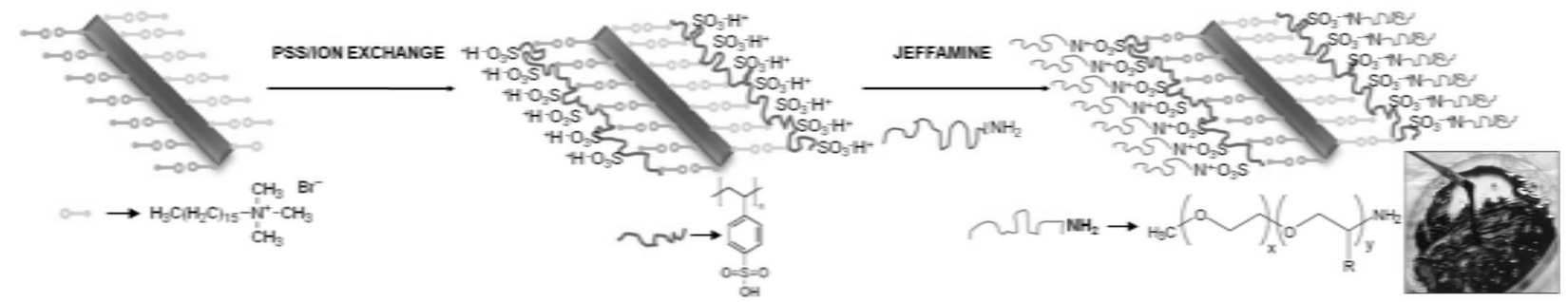

Scheme 1 

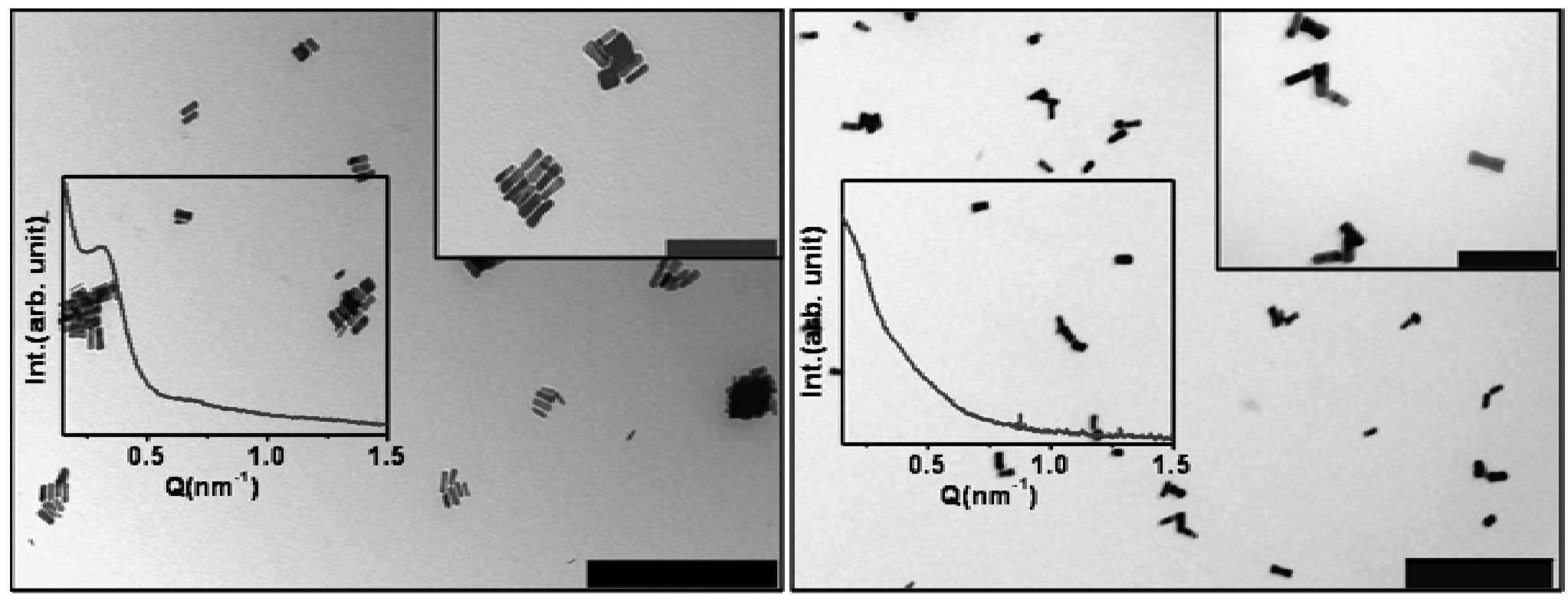

Figure 1 

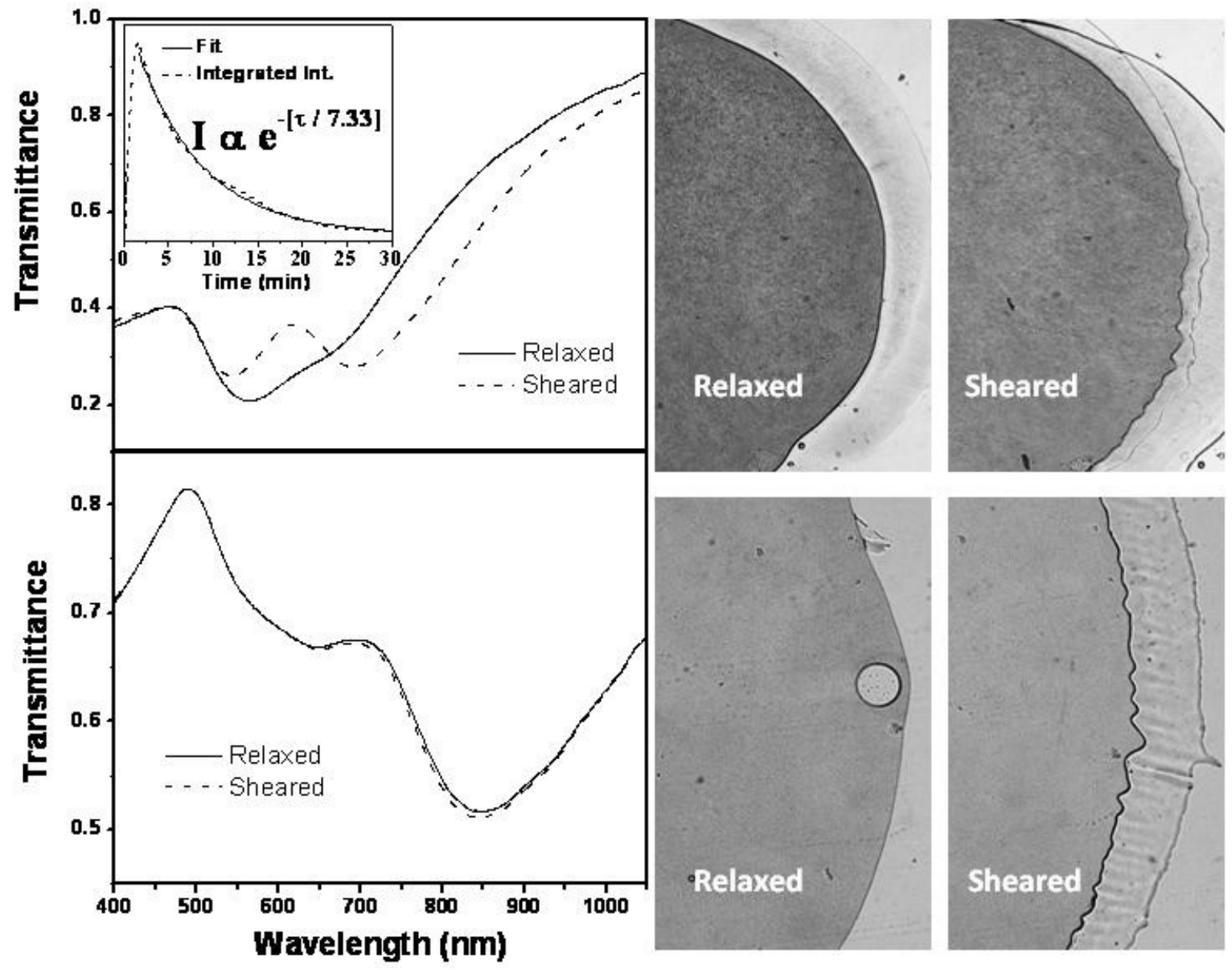

Figure 2 

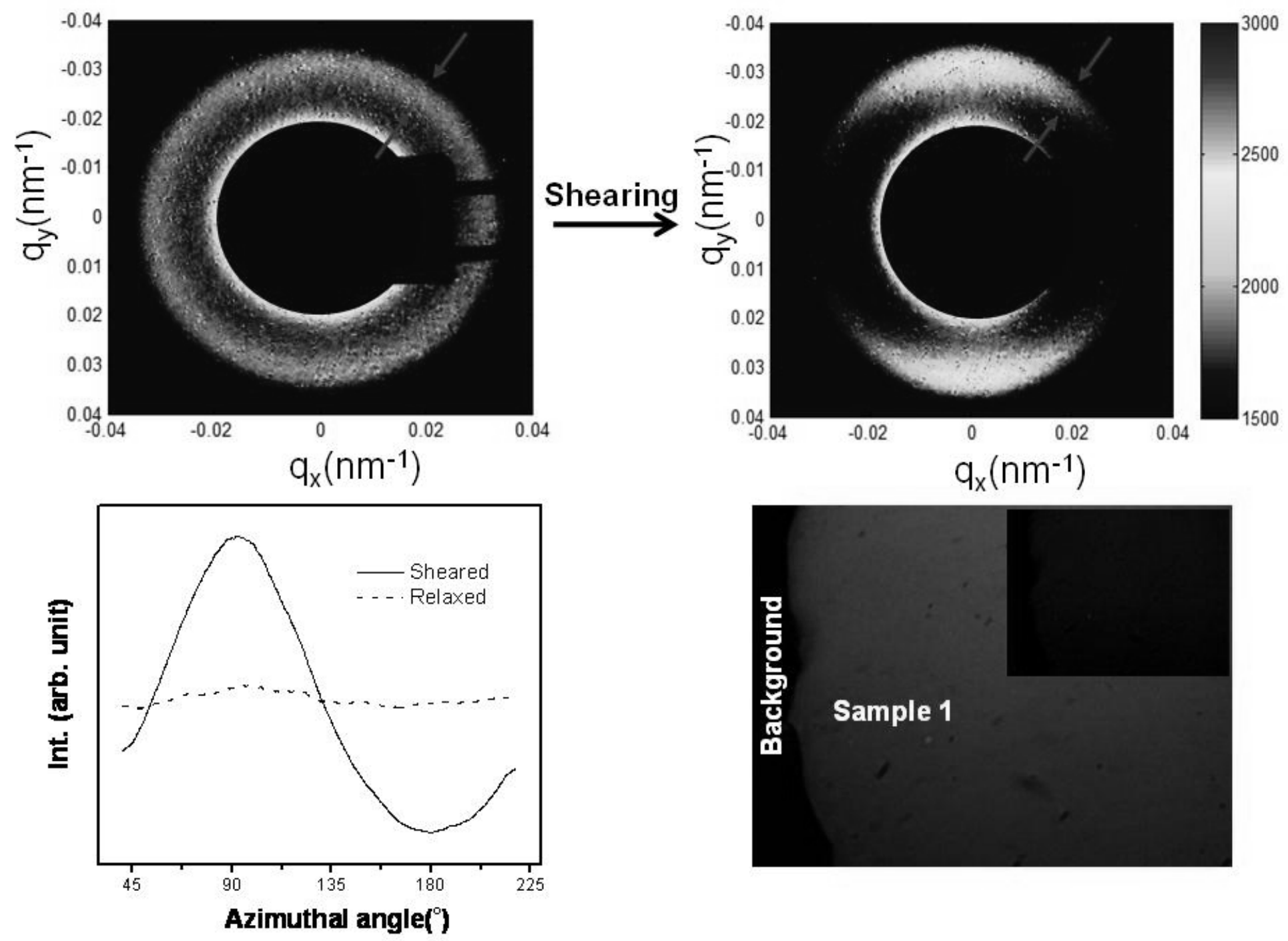

Figure 3 


\section{Supplementary Information}

\section{Solventless Gold Nanorods with Unique Optical Properties}

(A) Thermogravimetric analysis (TGA) data for samples 1 \& 2. (B) Video of dynamic color changes observed while shearing sample 1. (C) Video of dynamic spectral changes observed while shearing sample 1. (The video 'color change - 5x optical microscope.avi' of the dynamic color change observed on a non-polarized optical microscope and the video 'spectral change.avi' is not included in the manuscript and has been sent to the editorial office as separate attachments.)

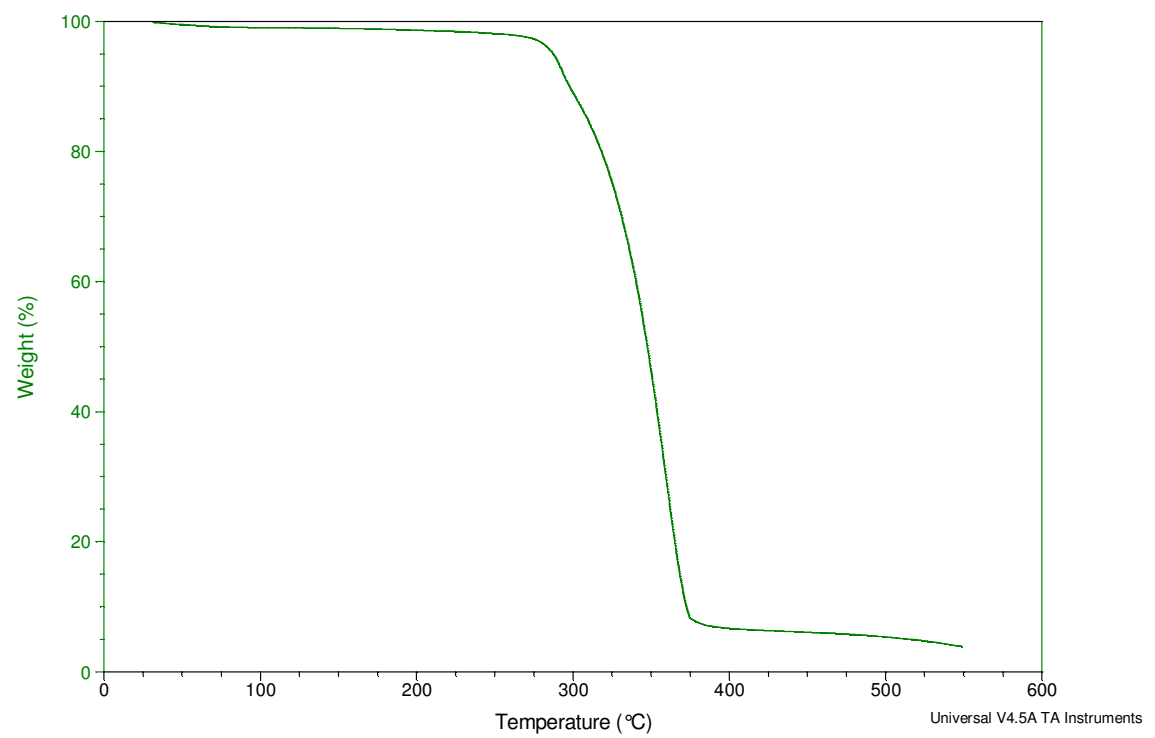

TGA data of sample 1.

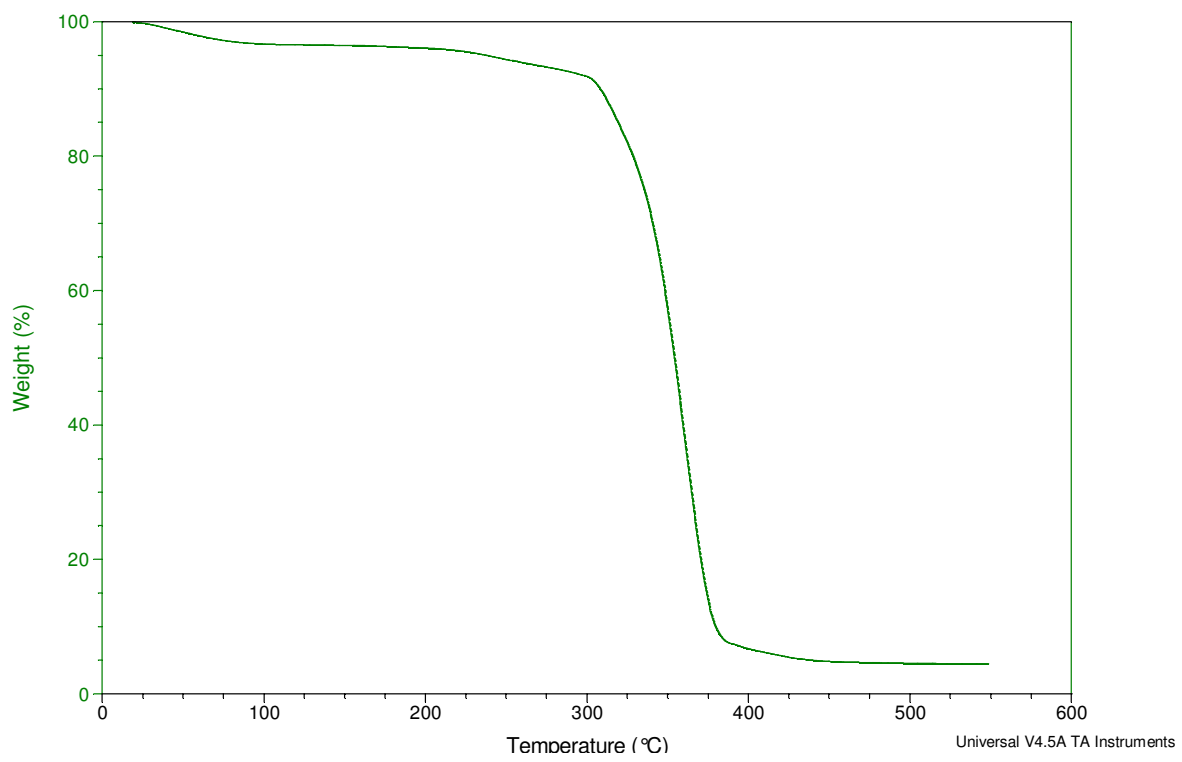

TGA data of sample 2. 
TOC graphic use only
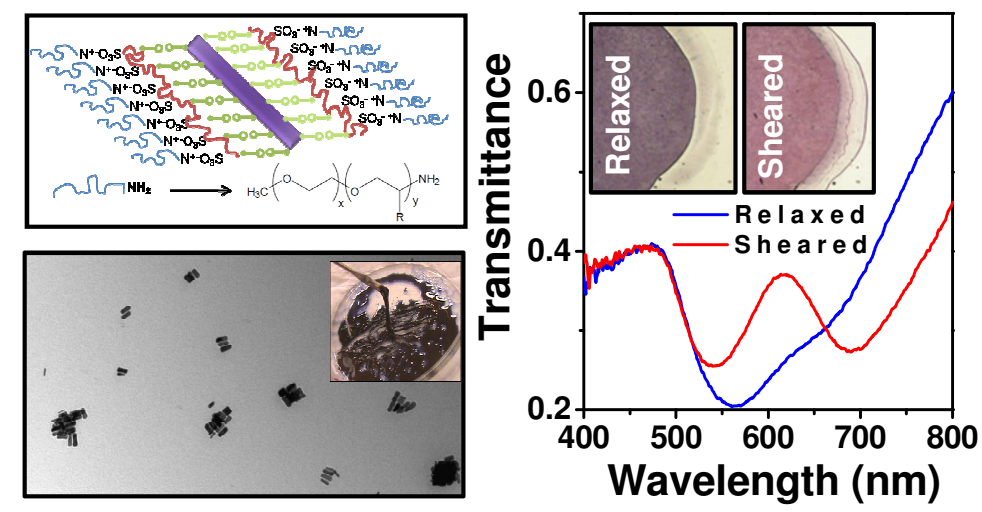